\title{
Bank Macro Safety Status Research Based on Robust MEWMA Process Control Method
}

\author{
Xie Xianfen ${ }^{1,4, a}$, Wang Binhui ${ }^{1, b} *$, Gu Wanrong ${ }^{2,4, c}$, Zhang Ziye ${ }^{3, d}$ \\ ${ }^{1}$ Jinan University, Guangzhou, 510632, China \\ ${ }^{2}$ South China Agricultural University, Guangzhou, 510642, China \\ ${ }^{3}$ South China University of Technology, Guangzhou, 510641, China \\ ${ }^{4}$ Guangdong Key Laboratory of Big Data Analysis and Processing, Guangzhou, 510006, China \\ axiexianfen@163.com, ’wbh100@126.com, 'guwanrong@scau.edu.cn, d472304094@qq.com \\ *Corresponding Author: Wang Binhui (wbh100@126.com)
}

Keywords: Bank Macro Safety Status; Robust Statistics; MEWMA; Process Control

\begin{abstract}
The issue of bank safety has always been one of the key contents of financial and economic scholars. First, this paper establishes the bank macro safety status index system, obtain robust covariance matrix based on the Fast-MCD algorithm. On this basis, construct robust process control model of the bank macro safety status, analyze bank macro safety's antecedent status, consistent status and the lagging status. The research shows that, the unsafe period of bank's macro safety status is mainly concentrated in the middle of 2005, end of 2008 to early 2009, end of 2011 to early 2012, which is basically consistent with China's actual financial situation. In addition, the antecedent status index is about 3 months ahead of the consistent index, and the lagging status index is roughly 6.3 months behind the consistent status index. These results can be used to predict the macro safety status of the bank and take the corresponding policy measures in advance.
\end{abstract}

\section{Introduction}

Scholars at home and abroad have always paid much attention to the study of bank safety, especially the outbreak of the Asian financial crisis in 1997-1998. The theory of banking crisis and the empirical research have received high attention and rapid development. Research on bank safety mainly focus on the construction of Safety Warning Index System, bank fragility and sensitivity analysis, financial risk measurement and so on. In the research work of constructing the security early warning index system, $\mathrm{Gu} \mathrm{Haidu}^{[1]}$ constructs the basic index of research from three dimensions: macro-economic operation, financial institution operation and external financial risk, for example, GDP growth rate, consumer price index growth rate and M2 growth rate. Then constructs the system of China financial security monitoring and evaluation index, determined the index weights on based on AHP method, and divided interval between Chinese financial safety zone. Finally use these to measure Chinese Financial Safety Index from 1995 to 2009 quantitatively. Jiang $\mathrm{Hai}^{[2]}$ selected 17 financial and economic indexes from the micro, macro and international markets, adopted empirical assignment and combined principal component analysis to determine indicators' weights, estimated China's financial security index for 1998-2007. Dai Zhimin et Al. ${ }^{[3]}$ conducted a study on the Early Warning System of bank safety, established a security warning index system based on management and Equity Control, Banking Control, Bank equity stability, credit control, risk contagion and financial supervision. Zhang Yan $^{[4]}$, Fan Jijun ${ }^{[5]}$ and Li Fangyan ${ }^{[6]}$ Carried out empirical research on the Financial Security Index and Chinese financial security early-warning index system.

In the aspect of vulnerability and sensitivity analysis, Wu Zhiwen ${ }^{[7]}$ has done some research on financial fragility; Wang Donghua ${ }^{[8]}$ analyzed the risk and sensitivity of the bank as a whole based on financial data of China's commercial banks and financial disclosure market. In the aspect of financial risk measurement, different scholars have used different methods to monitor the bank's safety status, Wan Xiaoli ${ }^{[9]}$ use specific risk type analysis method to measure the risk of Commercial 
Bank Banking System. Tian Haishan ${ }^{[10]}$ measured the systematic risk measurement of Chinese banks under the generalized hyperbolic distribution for the first time, and the results was more accurate than dynamical system risk measurement under $t$ distribution. Wang Yan et al. ${ }^{[11]}$ applied the extreme fractional regression to measure the financial risk under the assumption of the tail extreme distribution, and analyzed the influence factors of systemic risk contribution of financial institutions by using the panel regression analysis. Fan Hengdong ${ }^{[13]}$ used BP neural network to carry out the early-warning monitoring analysis of Chinese commercial banks, and Li Nan et al ${ }^{[14]}$ estimated the risk Var of Commercial Bank market based on semi-parametric estimation method of the pseudo-natural function of combination weight.

But the factors affecting the bank's macro-safety status are relatively single, and most of them use the econometric model. This paper establishes bank's macro-safety index system based on the index of bank's macro-safety status. Because outliers are inevitably exist in economic running, the existence of the outliers makes the results obtained by the traditional method incorrect. In this paper, we use the robust MEWMA control method to study the process control of the macro-safety status of Chinese banks, in order to accurately reflect the current state of macro-security of Chinese banks, which can provides decision-making for government departments.

\section{The overall approach to index system construction}

Our research is an analysis of the security of the banking system in the society as a whole, the bank safety index system should be constructed at macro level to cover the contents of bank capital adequacy, asset quality, liquidity, sensitivity to market risk and government support. In actual studies, some index may have incomplete data and some can be replaced with other comparable index or ratios. From the actual data available and other factors, the indexes selected in this paper include M1, Economic Consistent Index, Fiscal Deficit/GDP, CPI Growth, Fixed asset investment growth, Broad Currency/Foreign exchange reserve, Exchange rate, Stock market value/GDP, Bonds, Domestic net asset/M1, Net export, Bank foreign direct investment, Short-term foreign currency loans/Foreign exchange reserves, Bank domestic credit growth/GDP, etc.

Table 1 Bank macro safety status index systems

\begin{tabular}{c|l}
\hline & $X_{1}:$ Consistent Index \\
& $X_{2}:$ Currency M1 \\
Antecedent Index & $X_{3}$ : Fiscal Deficit / GDP \\
& $X_{4}$ : Domestic Credit Growth/ GDP \\
& $X_{5}:$ CPI Growth \\
& $X_{6}:$ Fixed Asset Investment Growth Rate \\
\hline & $X_{7}:$ Broad Currency / Foreign Reserves \\
& $X_{8}:$ Exchange Rate \\
Consistent Index & $X_{9}:$ Interbank Lending Weighted Interest Rate \\
& $X_{10}:$ Bonds \\
& $X_{11}$ Total Stock Market Price /GDP \\
& $X_{12}:$ Net Foreign Assets /M1 \\
\hline \multirow{5}{*}{ Lagging Index } & $X_{13}:$ Net Exports \\
& $X_{14}:$ Short-term Foreign Currency Loans / Foreign Reserves \\
& $X_{15}:$ Medium and Long-term Deposits / Medium and Long-term \\
& Loans \\
& $X_{16}:$ Foreign Direct Investment \\
\hline
\end{tabular}

\section{Robust MEWMA Process Control Chart}

Set the sample data matrix is $X_{n \times p}, \mu$ is overall mean vectors, $\Sigma$ is overall covariance Matrix, $x_{i}$ satisfies: $x_{i} \sim N_{p}(\mu, \Sigma), i=1,2, \cdots, n$, after the transformation of MEWMA, the data matrix is recorded 
as $W$. Its statistics are defined as:

$$
\left\{\begin{array}{l}
w_{0}=0 \\
w_{i}=R\left(x_{i}-\mu_{0}\right)+(I-R) w_{i-1}
\end{array}\right.
$$

Here, $w_{0}$ is the P-weft Zero Vector, $R=\operatorname{diag}\left(r_{1}, r_{2}, \cdots, r_{p}\right), \quad 0 \leq r_{j} \leq 1, j=1,2, \cdots, p, I$ is the P-weft Unit Matrix, $\mu_{0}$ is the mean vector of $x_{i}$ when the process is in a controlled state. Usually, let $r_{1}=r_{2}=\cdots r_{p}=r(0 \leq r \leq 1)$, these observations have the same weight, and now the statistics are:

$$
\left\{\begin{array}{l}
w_{0}=0 \\
w_{i}=r\left(x_{i}-\mu_{0}\right)+(I-r) w_{i-1}
\end{array}\right.
$$

Here, $r$ is a smoothing factor, and the closer it gets to 1 , the lower the weight assigned to the previous data, and when the value is 1 , the control chart is generally translated into a Hotelling's control chart.

Based on the preceding statistics, the observations can be defined as:

$$
Q_{i}=w_{i}^{\prime} \Sigma_{w_{i}}^{-1} w_{i}
$$

Here, $\Sigma_{w_{i}}$ is covariance matrix of $w_{i}$. And then iterate $w_{i}$ over and over. Until $i \rightarrow \infty$, we can get the asymptotic approach of $\Sigma_{w_{i}}$ :

$$
\Sigma_{w_{i}}=\left(\frac{r}{2-r}\right) \Sigma
$$

As you can see from the formula, just given the smoothness factor $r, \Sigma_{w_{i}}$ can be obtained by $\Sigma$. Then statistics of MEWMA control chart can be expressed as:

$$
Q_{i}=w_{i}^{\prime}\left(\frac{r}{2-r} \Sigma\right)^{-1} w_{i}=\left(\frac{r}{2-r}\right) w_{i}^{\prime} \Sigma^{-1} w_{i}
$$

Let UCL be the control limits to achieve a certain average running length ARL when in controlled status, and UCL $>0$. It should be noted that the control limit for the MEWMA control chart is different from that of the Hotelling $\mathrm{T}^{2}$ control chart, which cannot be directly computed. Rabhu and Runger(1997) used the Markoff chain method to study this problem deeply, given UCL values of the MEWMA control chart with different variable numbers and smoothing parameters. Once identified the UCL, the process can be monitored. When observations $Q_{i}>\mathrm{UCL}$, the MEWMA control chart will send a warning signal which indicates the process is out of control. When drawing the MEWMA control chart, the samples covariance Matrix $S$ is commonly used as the estimation of $\Sigma$ because the true value is unknown. It can be seen from above, that this statistic is not robust, and the covariance based on sample data has a great influence on the existence of the discrete group.

\section{Robust process control analysis of Bank macro-safety state}

\subsection{Data sources and basic descriptions statistical analysis}

The data from January 2003 to March 2012 used here are derived from the Chinese Statistical Yearbook, China macro-economic research database in the CSMAR database, the Chinese Education Yearbook and the Chinese Education Funding Yearbook., a total of 111 sample. Table 2 sets out the definitions of the variables and the basic descriptive statistical results.

As can be seen from the Table 2, most of the indicators have large standard deviations, some of which are larger than the absolute values of the mean, indicating that most of these indicators are non-normal. For example, the medium of Long-term Deposits/Medium and Long-term Loans is 1.1813, while the standard deviation is 9.627, far greater than the mean. It can be seen that the 
coefficient of variation of different index is different. The smaller coefficient of variation shows that variation of the index is small, and the larger coefficient of variation indicates that the index is more varied, and there may be some outliers, this will affect the bank macro-safety status, need to make further analysis. In addition, Skewness and Kurtosis values also suggest metrics are not subject to normal distributions.

Table 2 Definitions of the variables and the basic descriptive statistical results

\begin{tabular}{cl|ccccc}
\hline Variable & \multicolumn{1}{c|}{ Definitions } & Mean & SD & Cov & Kurtosis & Skewness \\
\hline $\mathrm{X}_{1}$ & Consistent Index & 101.07 & 2.156 & 0.021 & 4.88 & -1.440 \\
$\mathrm{X}_{2}$ & Currency M1 & 155.82 & 6812 & 0.437 & 1.91 & 0.560 \\
$\mathrm{X}_{3}$ & Fiscal Deficit / GDP & -2.037 & 5.037 & -2.473 & 6.74 & -1.045 \\
$\mathrm{X}_{4}$ & Domestic Credit Growth/ GDP & 12.69 & 14.82 & 1.168 & 11.49 & 2.522 \\
$\mathrm{X}_{5}$ & CPI Growth & 3.029 & 2.417 & 0.798 & 2.50 & 0.181 \\
$\mathrm{X}_{6}$ & Fixed Asset Investment Growth Rate & 10.64 & 12.38 & 1.164 & 2.20 & -0.051 \\
$\mathrm{X}_{7}$ & Broad Currency / Foreign Reserves & 34.211 & 11.40 & 0.333 & 2.90 & 1.101 \\
$\mathrm{X}_{8}$ & Exchange Rate & 7.477 & 0.747 & 0.100 & 2.07 & 0.097 \\
$\mathrm{X}_{9}$ & Interbank Lending Weighted Interest & 2.187 & 0.762 & 0.348 & 3.78 & 0.698 \\
$\mathrm{X}_{10}$ & Rate & 34.92 & 20.46 & 0.586 & 2.12 & 0.534 \\
$\mathrm{X}_{11}$ & Total Stock Market Price /GDP & 3.225 & 2.542 & 0.788 & 5.50 & 1.563 \\
$\mathrm{X}_{12}$ & Net Foreign Assets /M1 & 0.014 & 0.017 & 1.225 & 23.9 & 1.315 \\
$\mathrm{X}_{13}$ & Net Exports & -131.9 & 101.6 & -0.770 & 2.84 & -0.363 \\
$\mathrm{X}_{14}$ & Short-term Foreign Currency Loans / & 0.5815 & 0.295 & 0.508 & 2.10 & 0.619 \\
& Foreign Reserves & & & & & \\
$\mathrm{X}_{15}$ & Medium and Long-term Deposits / & 1.1813 & 9.627 & 8.149 & 49.01 & 2.356 \\
$\mathrm{X}_{16}$ & Medium and Long-term Loans & & & & & \\
\hline
\end{tabular}

\subsection{Bank marco-safety status analysis}

Due to bank macro-safety index system including the antecedent indicator group, the consistent index group and the lagging indicator group, the macro-safety status of banks will be analyzed from these three aspects. Through the multi-variable process monitoring of the antecedent indicator group, it is possible to predict the future macro-safety operation state of the bank, and monitor the process of consistent index group, monitoring of the lagging indicator group can be used to confirm the bank's macro-safety status. According to the Fast-MCD algorithm, calculate the mean vectors $\bar{X}$ and covariance matrix of the three samples, and substitute into statistical calculation formula of MEWMA control chart. Here, smoothness factor $\lambda=0.4$, confidence interval $\alpha=0.01$, dimensions of these three samples are $p=6,6,4$. These three status MEWMA control chart are list from Fig. 1 to Fig.3.

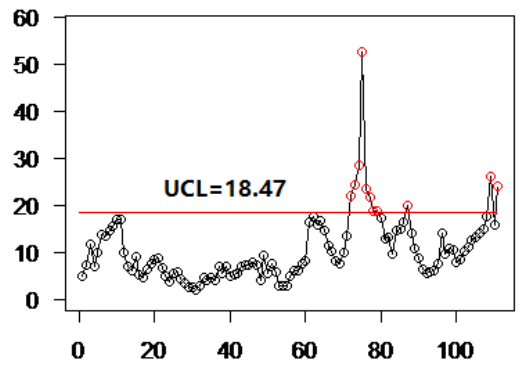

Fig.1 Antecedent status

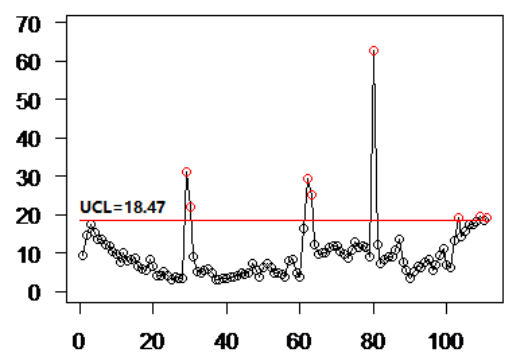

Fig.2 Consistent status

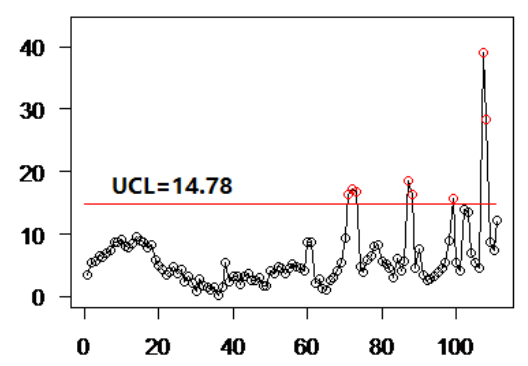

Fig.3 Lagging status

As can be seen in Fig.1, a total of 11 points fall outside the control limit and can be considered as outliers, as follows:2008-12, 2009-01, 2009-02, 2009-03, 2009-04, 2009-05, 2009-06, 2009-07, 
2010-03, 2012-01, 2012-03.In Fig.2, there are 8 points fall outside the control limit, as follows: 2005-05, 2005-06, 2008-03, 2008-04, 2009-08, 2011-07, 2012-01, 2012-03.There are also 8 outliers in Fig.3:2008-11, 2008-12, 2009-01, 2010-03, 2010-04, 2011-03, 2011-11, 2011-12

From above three types process control chart of banks macro-safety status, we can see that, the outliers falling outside the control limit have a certain degree of concentration and continuity. It can also be seen that volatility of these three indexes is regular, and there is a corresponding relationship between these three kinds of indexes. Based on these principles, with the exception of a few discrete outliers, can divided them into five time periods, as shown in Table 3.

Table 3 Classification tables for the three types of indicators

\begin{tabular}{cccc}
\hline & Antecedent indexes & Consistent indexes & Lagging indexes \\
\hline Time 1 & & $2005-05$ to $2005-06$ & \\
Time 2 & & $2008-03$ to $2008-04$ & $2008-11$ to 2009-01 \\
Time 3 & 2008-12 to 2009-07 & $2009-08$ & $2010-03$ to 2010-04 \\
Time 4 & & $2011-07$ & $2011-11$ to 2011-12 \\
Time 5 & 2012-01 to 2012-03 & 2012-01 to 2012-03 & - \\
\hline
\end{tabular}

First, the paper analyzes the outliers' correspondence between antecedent indexes and consistent indexes. As you can see, at Time 3 the antecedent indicator is roughly 6 months ahead of the consistent one; in Time 5, the antecedent and consistent indicators have the same outlier time. In the other three time periods, the outliers appeared in the consistent index group, but there was no outlier value in the antecedent index group, the specific reason needed further analysis. Overall, the antecedent indicator averaged a consensus indicator for three months over those five time periods. Then, we analyze the outliers corresponding to the state of the consistent index and the lagging index. In Time 2, the antecedent indicator state lags behind the consistent target for 8 months; in Time 3, the lagging indicator is seven months behind the consistent target; and in the time period 4, the lagging indicator falls behind the consistent target for 4 months. Overall, the lagging indicator was substantially behind the consistent target of 6.3 months.

\section{Summary}

Based on the analysis above, the following characteristics can be seen: (1) during the period from 2003 to 2012, the bank macro-safety has experienced from high risk to low risk, and then from low risk to high risk, which may exist some periodicity. (2) MEWMA process control chart of the consistent status indexes identified five periods of insecurity, which were largely concentrated in three phases: middle of 2005, end of 2008-early 2009, end of 2011-early 2012. (3) In 2005, China introduced a series of reform measures in the banking field. The reform of the state-owned commercial banks in China is at a special period, and the reform of the shareholding system of Bank of China and China Construction Bank is also in progress, however, because the reform of the banking supervision system is not a short-term matter, the laws and regulations need to be perfect, so the inconsistency between the backward state and the opening speed of the banking supervision system has also caused the decline of bank safety. (4)2008 was the second trough of the study period, mainly due to the United States subprime mortgage crisis of the world economy and the adoption of quantitative easing by the Fed, which has further flooded global liquidity.

In this paper, we use China's monthly data of 2003-2012 and Fast-MCD algorithm to establish a robust process control chart of bank's macro-safety. The state index value of the antecedent indicator can be used to predict the state of the bank's macroscopic operation outside the control limit. The trend of Bank macro-security state obtained by the above-mentioned research is basically in line with the actual financial security situation in China. If the state curve of the leading index is out of group, then the probability of real outliers in the next 3-6 months is very high, at this time, we should carry on the comprehensive analysis to the bank macroscopic security state and take corresponding policy measures ahead of time. 


\section{Acknowledgements}

This work was financially supported by General projects of the National Fund for Social Sciences (16BTJ035), Guangdong Natural Science Foundation Project (2018A030313437) and the open fund project of Guangdong Key Laboratory of Big Data Analysis and Processing (2017006, 2017010).

\section{References}

[1] Gu Haibing, Zhang Anjun. A comparative analysis of the Dynamic Monitoring of China's Financial Security Index [j]. Forum in Fujian, 2012, 3:11-17

[2] Jiang Hai. The estimation and Empirical Study of China's Financial Security Index: 1998-2007[J]. Contemporary Finance, 2009, 10:47-53

[3] Dai chi-min. Research on bank safety warning system based on foreign bank's participation [J]. Journal of Zhejiang University (humanities and Social Sciences), 2009, 39(5):19-26

[4] Zhang Yan. A review of the literature on the risk evaluation index of Commercial Banks [J].2013, 16:146-168

[5] Fan ka-jun. Construction and empirical analysis of Risk Early Warning System [J]. The modern economy, 2013, 19:104-106

[6] Li Fangyan. Research on risk evaluation of commercial banks in China [D], 2014:17-20

[7] Wu Zhiwen. Financial Integration and Financial Fragility: cross-country Comparative Study [J]. Economic Science, 2008, 6:78-90

[8] Wang Donghua. Risk Measurement and sensitivity analysis of commercial banks in china-based on our commercial bank financial data and Financial Market Open Data [j]. Systems engineering theory and practice, 2013, 33(2):284-295

[9] Wan xiao-li. The judgment and measurement of the fragility of the financial system in China 1987-2006[J]. Financial Research, 2008, 6:80-93

[10] Tian Haishan, Zhou Yuqin, Wu Hengyu. Study on dynamic systemic risk in generalized hyperbolic distributed families [J]. Mathematical Statistics and Management, 2017(1):59-72.

[11] Wang Yan, Chan soo-dong. Analysis of systemic financial risk and its influencing factors under the extremum distribution [j]. Mathematical Statistics and Management, 2014, 33(6):1011-1020

[12] Fan hang-dong. Research on risk early warning of China's commercial banking system based on neural network model [D], 2013:23-29

[13] Li Nan, Fan Pengying, Wang Yan, Min Chen. Research on market risk of commercial banks based on semi-parametric estimation under new capital agreement [J]. Mathematical Statistics and Management, 2014, 33(6):1080-1089 